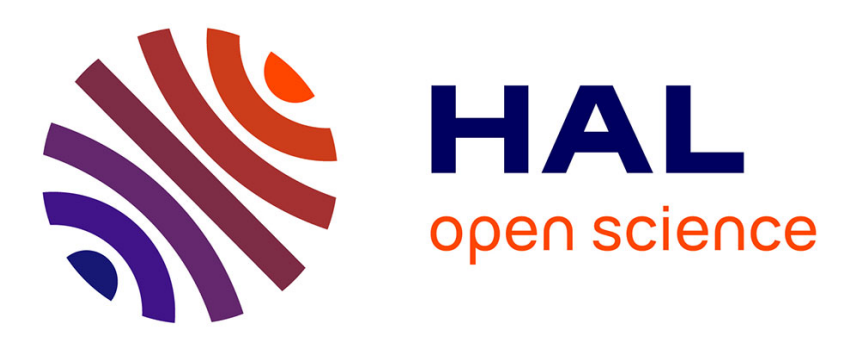

\title{
Anna, un sujet en quête d'asile. Les effets psychiques du système politique sur le réfugié et son thérapeute
}

Elise Pestre

\section{To cite this version:}

Elise Pestre. Anna, un sujet en quête d'asile. Les effets psychiques du système politique sur le réfugié et son thérapeute. L'Information Psychiatrique, 2008, Biopolitique, 6 (84), pp.569-576. 10.3917/inpsy.8406.0569 . hal-01508511

\section{HAL Id: hal-01508511 \\ https://hal.science/hal-01508511}

Submitted on 20 Jun 2017

HAL is a multi-disciplinary open access archive for the deposit and dissemination of scientific research documents, whether they are published or not. The documents may come from teaching and research institutions in France or abroad, or from public or private research centers.
L'archive ouverte pluridisciplinaire HAL, est destinée au dépôt et à la diffusion de documents scientifiques de niveau recherche, publiés ou non, émanant des établissements d'enseignement et de recherche français ou étrangers, des laboratoires publics ou privés. 


\title{
Anna, un sujet en quête d'asile. Les effets psychiques du système politique sur le réfugié et son thérapeute
}

\author{
Élise Pestre
}

\begin{abstract}
RÉSUMÉ
Les politiques gouvernementales qui mènent aujourd'hui un véritable combat contre l'immigration dite « clandestine » tentent de définir si le demandeur d'asile est un « vrai » ou un «faux » réfugié. Les incidences subjectives de ces soupçons seront envisagées à partir d'une rencontre singulière avec une jeune demandeuse d'asile. Le travail de reconstruction narrative engagé auprès d'elle permettra de penser la manière dont le sujet est par le truchement de son récit des persécutions destiné aux institutions de l'État, est affecté en profondeur par les traumatismes. Cette relation transférentielle mettra en lumière que celui qui écoute ce qui touche à la cruauté humaine, en l'occurrence le psychothérapeute, n'est pas indemne de cette violence.
\end{abstract}

Mots clés : renarration, transfert, réfugié, traumatisme, témoignage

\section{ABSTRACT}

Anna, a person looking for asylum. The psychological impact of the political system on refugee and therapist. Government policies today tend to define asylum seekers as "real" or "fake" refugees. They are conducting a veritable campaign against immigration that they label "clandestine". In this article the subjective effects of such suspicions will be explored based on a particular case of a young female asylum seeker. The task of narrative reconstruction undertaken enables the analysis of how profoundly traumatised the asylum seeker is by describing the persecution she has suffered to state agencies. The analysis of this transferential process illuminates how the person listening, in this case the psychotherapist, can not be left unscathed by what amounts to human cruelty.

Key words: refugee, trauma, testimony, narration, transference

\section{RESUMEN}

Anna, un sujeto en busca de asilo. Los efectos psíquicos del sistema político sobre el refugiado y su terapeuta. Las opciones políticas gubernamentales que entablan un verdadero combate contra la inmigración llamada "clandestina", intentan averiguar si la persona que pide el asilo es un "verdadero" o un "falso" refugiado. El artículo analiza las incidencias subjetivas de estas sospechas a partir del encuentro singular con una joven que pide el asilo. El trabajo de reconstrucción narrativa permitirá pensar la manera en que el sujeto, a través del relato de sus persecuciones destinado a las instituciones del Estado, se ve afectado profundamente por los traumatismos. Esta relación transferencial pone en evidencia la manera en que, el que escucha lo que concierne la crueldad humana, en este caso el psicoterapeuta, no puede quedar indemne frente a esta violencia.

Palabras clave : renarración, transferencia, refugiado, traumatismo, testimonio

CRPM, université Paris-VII-Denis-Diderot, 41, rue Sedaine, 75011 Paris

<elise.pestre@gmail.com>

$\overline{\text { Tirés à part : } E}$. Pestre 
Les sujets en quête de refuge doivent produire aux autorités de l'État français, et dans la langue du pays d'accueil, un récit qui retrace les faits vécus et la fuite des persécutions ${ }^{1}$. Aux difficultés rencontrées pour s'exprimer dans une langue autre que la sienne et tenter de se plier aux règles d'une rhétorique inconnue, se déploie chez ce narrateur la tentative de répondre à l'impératif juridique en jeu : « tout raconter ». En effet, la remémoration des événements devient un absolu auquel le sujet se doit de répondre dans l'urgence - les délais accordés sont désormais très brefs. Pourtant, ce procédé est intimement soumis aux aléas de la subjectivité, car se souvenir, reconstituer avec précision des événements, est une opération qui traverse les différents systèmes mémoriels, les registres Conscient, Pré-conscient et Inconscient, et qui demande une certaine disposition psychique.

C'est à partir d'une rencontre clinique avec une jeune demandeuse d'asile, et la rencontre est ici à entendre dans le sens « d'une mise en présence fortuite ou non de deux éléments qui vont avoir une action l'un par rapport à l'autre [9] », que je proposerai des hypothèses concernant les effets de la « machinerie juridique » propre à l'État sur le sujet. Seront aussi envisagés les effets multiples et inattendus relatifs à cette clinique extrême sur le thérapeute.

\section{Une rencontre singulière}

Je rencontrai Anna, une jeune femme d'Afrique centrale âgée de vingt-trois ans, au sein d'une association d'aide juridique aux migrants où j'avais créé une consultation psychologique. C'est dans le cadre de la réouverture de son dossier à l'Ofpra ${ }^{2}$ qu'elle consultait. Le réexamen de son dossier par l'administration impliquait une nouvelle narration de son parcours de réfugiée jusqu'en France ainsi qu'une audition auprès d'un fonctionnaire. La jeune femme confia à l'un des membres de l'association que cette audition à venir "l'inquiétait beaucoup », ce réexamen étant sa "dernière chance » avant qu'elle soit définitivement déboutée du droit d'asile ${ }^{3}$. En tant que psychologue de l'association, je lui proposai de me rencontrer afin qu'elle évoque ses inquiétudes face à cette nouvelle demande de statut de réfugié ; Anna accepta l'entretien.

Pourquoi ne bénéficiait-elle pas de ce statut, alors même qu'elle entrait précisément dans le cadre de la Convention

\footnotetext{
${ }^{1}$ Les demandeurs d'asile qui ne parlent pas français doivent payer un traducteur à leurs frais pour que soit traduit leur récit en français. Mais ceux qui sont francophones renoncent généralement au traducteur et tentent d'écrire eux-mêmes leur texte.

${ }^{2}$ L'Ofpra (Office français pour réfugiés et apatrides) et la CRR (Commission de recours aux réfugiés) sont les deux instances qui ont en charge la délibération du statut de réfugié.

${ }^{3}$ Il est possible d'intenter un pourvoi en cassation contre la décision de la Commission des recours aux réfugiés, mais ce recours reste peu usité. Pour plus de précisions consulter Le Guide de l'entrée et du séjour des étrangers en France. Paris : Gisti, Syros, 2000, p.207.
}

de Genève ? Les motifs de refus des organes décisionnels semblaient «incompréhensibles et insensés » aux juristes de l'association. En effet, tous les proches d'Anna avaient disparu - ses parents ainsi que ses sept frères et sœurs avaient été tués -, et elle n'avait «plus rien ni personne là-bas », comme elle-même le formulait ${ }^{4}$. Elle était francophone, logée dans un foyer pour demandeurs d'asile ${ }^{5}$, et suivait une formation. En un mot, elle avait le «profil idéal » pour obtenir la qualité de réfugié.

Bien que l'aide au récit ne soit pas la vocation de l'association, je me sentis rapidement happée par la tragédie de cette jeune femme, l'urgence de sa demande, et l'« élection » qu'elle témoignait à mon égard. Je lui proposai que nous entreprenions ensemble cette remise en récit exigée par l'État. Si sa demande manifeste résidait dans l'obtention de la qualité de réfugié, je supposais qu'une demande plus latente, adressée à la psychologue que je suis, existait.

Tisser ensemble et progressivement «quelque chose » de son vécu lié à la fuite des persécutions lui permettrait d'historiciser son vécu, lui disais-je, qu'elle accède ou non à ce statut. Elle accepta cette proposition.

Anna suivait une psychothérapie au sein d'un centre de soins destiné aux demandeurs d'asile. Et si elle « se sentait un peu mieux » depuis qu'elle avait débuté ce travail, me confia-t-elle, certains symptômes s'étaient pourtant accentués depuis qu'elle allait "redemander l'asile ». Sa souffrance liée à ses troubles psychotraumatiques lui «causait $d u$ tort » dans sa vie quotidienne. Elle évoqua ses douleurs physiques (migraines persistantes et problèmes de digestion), le fait qu'elle ait toujours des «crises d'angoisse », qu'elle se sente triste et qu'elle fasse des cauchemars. Mais c'est surtout « ses oublis » et « son amnésie » qui la préoccupaient : "C'est horrible parce que j'ai comme des trous dans la tête. J'ai oublié plein de choses. "Symptômes envahissants qui empêchaient le déroulement construit de son récit.

Le « contrat » établi entre nous était le suivant : nous nous verrions le temps nécessaire à la relecture et au remaniement de son texte, à raison d'une fois par semaine.

Je m'aperçus ensuite à quel point la rapidité de son investissement transférentiel et son imbrication à mon propre engagement transférentiel ${ }^{6} \mathrm{~m}$ 'amenèrent à donner une réponse immédiate, symptomatique, à sa demande [7].

\footnotetext{
${ }^{4}$ Toutes les paroles d'Anna sont rapportées en italique.

${ }^{5}$ Les Cada (Centres d'accueil pour demandeurs d'asile) sont accessibles à une minorité de demandeurs d'asile, les places étant rares. On sait que les réfugiés logés dans ces foyers ont plus de chances d'obtenir le statut de réfugié que ceux qui n'y vivent pas, dans la mesure où ils sont « pris en charge»à divers niveaux (social et juridique notamment).

${ }^{6}$ Nous préférons parler en termes de transfert que de contre-transfert chez le clinicien, comme G. Devereux a pu le proposer dans son ouvrage De l'angoisse de la méthode dans les sciences du comportement. Paris : Flammarion, « Nouvelle bibliothèque scientifique », 1980, p.75. « Le transfert et le contre-transfert ont des sources et des structures identiques. C'est stricte affaire de convention que d'appeler "transfert" les réactions
} 
Cette relation cristallisait les problématiques en jeu dans cette clinique, phénomènes ici particulièrement visibles et qui seront envisagés.

La supervision que je débutais me permit de comprendre quelque chose de cette position adoptée dans l'urgence et d'accéder à un étayage théorico-clinique inexistant au sein de l'association. Je retracerai l'évolution du travail de réécriture entrepris avec Anna qui, s'il se prolongea sur une période de six mois, parvint malgré tout à se dégager d'une finalité juridico-sociale.

Lors de notre deuxième entrevue, Anna me tendit d'emblée son témoignage écrit. Au vu de la rigidité de son corps et de la distance qu'elle manifestait, je n'osai pas lui demander de m'en faire la lecture. Je parcourus ce récit, dont la calligraphie exprimait déjà beaucoup en soi : les mots s'entrechoquaient, les ratures étaient insistantes. Lors de ma lecture, je ressentis l'attention soutenue d'Anna à mon égard ; c'était comme si elle « guettait » en moi les réactions et les affects qui pourraient transparaître ou s'échapper de mon corps. Mes émotions à vif, difficilement dissimulables, et perçues par la jeune femme, lui permettaient sans doute d'entrevoir l'effroi qu'éveillait chez un autre son témoignage. La découverte des effets de son vécu de terreur sur son interlocuteur venait probablement valider chez elle la « certification » qu'un passé « extraordinaire » avait bien eu lieu. En effet, "l'expérience qu'il [le survivant] relate est si incroyable que, par moments, il doute de sa réalité ", écrit $\mathrm{R}$. Waintrater à propos des survivants de génocides [2, 13]. Le rescapé aurait-il besoin de voir chez les autres les réactions qu'il ne peut parfois pas éprouver lui-même. Mes affects semblaient venir compenser les siens, violents et dépressifs encore suspendus et déliés.

Après-coup, je m'aperçus combien j'avais été sidérée par ce témoignage. Si le récit lui-même m'avait terrifiée, la situation d'entretien m'avait elle aussi ébranlée : en lisant ce texte devant elle, spectatrice de mon propre effroi, mon malaise s'était amplifié. Je me sentis envahie d'affects puissants, prise dans un corps à corps tenace avec la chair de ses mots. Des manifestations corporelles intenses allant d'une sensation d'oppression à celle d'étouffement me traversèrent. Lorsqu'une violence sans nom attaque la pensée et le corps de celui qui l'a subie, elle vient aussi frapper ceux de l'interlocuteur qui écoute ce type de récit.

Après cette lecture, je restai silencieuse. Je parvins malgré tout à sortir de ma torpeur pour formuler à Anna combien il avait dû être difficile pour elle de survivre à cette tragédie et trouver des mots pour relater son vécu.

de l'informateur ou de l'analysé, “contre-transfert" celle de l'ethnologue ou de l'analyste. "

\section{Le contenu du récit destiné à l'État}

Les exactions débutèrent lorsque Anna, âgée de douze ans, était en vacances chez sa tante. Dès le premier jour, avec sa tante et son cousin, ils parvinrent à se cacher dans la maison. Puis, après être restés enfermés des semaines, ils

s'échappèrent, l'objectif étant d'atteindre les camps de réfugiés. Ils marchèrent pendant des mois au côté d'autres persécutés, se cachant la journée et poursuivant leur route la nuit pour échapper aux milices. Alors que les maladies tuaient les uns, les autres mouraient d'épuisement, de faim ou encore de déshydratation. Dans ce chaos, Anna perdit sa tante et son petit-cousin, et apprit plus tard, en France, qu'ils étaient décédés du choléra dans un camp de réfugiés.

Comme les pilonnages des camps étaient incessants, Anna reprenait quotidiennement la fuite avec des centaines d'autres. Trois années passèrent. Âgée de quinze ans, seule dans son errance, elle relate comment, « avec la famine, la fatigue et le fait que les femmes et les filles étaient presque nues, les hommes devenaient comme des bêtes sauvages ». Une nuit, on tenta de la violer ; un inconnu allongé à proximité d'elle la «sauva».

Cet homme, Jérôme, l'accompagna jusqu'à un camp de réfugiés où ils restèrent ensemble plusieurs mois. En raison du climat d'insécurité insupportable (recrutement obligatoire des hommes pour le combat, viol des femmes...), ils se résolurent à fuir pour l'Europe. Aidés par une connaissance de son compagnon, ils partirent en France où ils déposèrent chacun une demande d'asile.

\section{Symptomatologie psychotraumatique : oublis, « incohérences » et retour du refoulé}

Dès la première lecture du témoignage d'Anna, le déroulement de sa fuite jusqu'en France m'apparut bousculé, voire détemporalisé. Confrontée à une rupture de la cohérence narrative, je me sentais non seulement perdue dans les méandres du récit de sa survivance mais également égarée dans les «trouées »spatio-temporelles qui ponctuaient sa narration [10]. Il semblait que de longues périodes avaient été évacuées de son récit. De son errance prolongée au cœur de la forêt, Anna conservait peu de souvenirs et ne parvenait pas, me dit-elle, à se rappeler des noms de villes traversées ni de certaines dates importantes demandées par les institutions de l'État qui sont celles qui délibèrent sur l'octroi du statut de réfugié.

Par ailleurs, des éléments contradictoires apparaissaient notamment quand Anna parlait de son compagnon. Elle le désignait en effet tantôt comme son mari, tantôt comme un ami. J'envisagerai ensuite la manière dont cette « apparente 
variabilité $»^{7}$, oscillation que l'on peut relier à sa souffrance psychique, témoigne de la ténacité des blessures traumatiques du survivant.

$\mathrm{Si}$, en tant que demandeur d'asile, elle était soumise par les autorités de l'État à l'impératif de « tout relater », la remémoration endiguée barrait bien la possibilité de se rappeler de façon exhaustive cette période chaotique. L'accès à certains événements était psychiquement oblitéré, et Anna ne parvenait pas à les penser. Ce « devoir de vérité » requis par les services administratifs suscitait chez elle un intense effort de rassemblement mémoriel, alors que justement un travail de protection à l'égard du psychisme assailli par l'expérience traumatique avait eu des effets sur sa mémoire devenue défaillante. Et là se situait bien le cœur de la problématique rencontrée par les demandeurs d'asile : comment se remémorer l'impossible dans l'urgence d'un impératif qui exige une véridicité absolue ? Les scènes verrouillées par l'emprise traumatique rendaient la mise en récit forcément lacunaire, fragmentée, loin de la précision requise par les autorités décisionnelles.

\section{Un récit à plusieurs voix}

Anna m'expliqua, lors de l'entretien suivant, que son premier récit rejeté «n'était pas le sien ». Si le témoignage formulé devant la Commission de recours aux réfugiés relatait en partie son vécu, il était entrecoupé par des bribes de celui de Jérôme. "Sous ses ordres », mais aussi en raison de sa profonde angoisse, Anna avait narré un texte qui n'avait convaincu ni l'agent de protection (de l'Ofpra) ni les juges (de la CRR). Aussi avait-elle intégré à son propre témoignage des éléments de l'histoire de son compagnon et d'autres faits, fictifs. Ses déclarations initiales étaient donc, et comme pour beaucoup de réfugiés, multiples et contradictoires, en partie siennes et en partie autres [12].

Anna me conta ensuite comment Jérôme disparut à l'annonce du rejet à sa demande d'asile : "Il est devenu comme fou et il est parti tenter sa chance dans un autre pays européen. » Elle parlait de cette fuite en disant : "Il $m$ 'a abandonnée. » Je compris peu à peu que si cet homme l'avait au départ sauvée d'un viol, il l'avait ensuite contrainte, pour continuer à la « protéger », à avoir des relations sexuelles avec lui. Son chantage et ses menaces insidieuses vécues durant des mois émergeaient à travers le discours voilé de la jeune femme. Afin qu'il reste auprès d'elle, Anna avait dû répondre à ses « avances ». L'apport de cette «pièce manquante » me permit d'appréhender différemment son histoire et d'entendre quelque chose de ce qui s'articulait à sa trajectoire traumatique [5].

Ces éléments, qu'elle introduisait par fragments au fil de nos rencontres et qui concernaient sa vie intime, étaient difficiles à mettre en mots par la jeune femme qui ne voulait "surtout pas lui faire de reproches », cet homme lui ayant "sauvé la vie ». Elle ressentait ainsi une forte ambivalence à son égard : à la reconnaissance se mêlaient des sentiments de peur, de honte et de dégoût.

Je saisis progressivement les raisons pour lesquelles il était si complexe pour Anna de composer autour de cette relation et de parler, dans son témoignage, à la première personne. Elle éprouvait de la culpabilité à prononcer des accusations contre Jérôme, et pour cette raison même, ne parvenait pas à éclaircir cette histoire aux instances de l'État. Cette situation de menace permettait d'appréhender, en partie, les raisons pour lesquelles son premier récit n'était pas tout à fait le sien. Et pourtant, sans l'énoncé de cet élément déterminant expliquant son attitude première à l'égard de l'institution, elle serait soupçonnée de mentir à nouveau. On se situait bien au cœur de la problématique rencontrée par nombre de réfugiés : dire à l'autre ce que l'on doit taire à soi-même.

\section{Transfert et émergence de la névrose infantile}

Au fil de nos entrevues et avec ses souffrances peu à peu déposées, l'espace transférentiel se déployait. Et avec le travail de « renarration ${ }^{8}$ propre à l'écriture, les paroles et les maux se déliaient. La jeune réfugiée inscrite dans un travail de reconstruction narrative associait auprès de moi, autour de son "empêchement à parler de sa relation amoureuse » avec son psychothérapeute : "C'est un psychologue, oui... mais c'est un homme ».

Peu après avoir évoqué sa relation à son psychothérapeute, émergea un souvenir infantile. À huit ans, un oncle maternel qu' « elle aimait beaucoup » et de passage dans la famille, l'avait " touchée une nuit, alors que tout le monde dormait ». Avec sa tentative de rassemblement mémoriel

lié aux événements récents et très violents, la survenue d'autres souvenirs anciens qui s'imposaient avec force surgissait. Dans quelle mesure l' « incohérence » de son premier récit, selon la terminologie de l'administration, n'avait-elle pas été « parasitée » par ce souvenir infantile?

Je proposai l'interprétation suivante à Anna : ce dégoût et sa culpabilité ressentis à l'égard de Jérôme se mêlaient probablement au souvenir de cet événement où son oncle, un homme qu'elle « aimait », et en qui elle avait confiance, avait lui aussi abusé d'elle. À la suite de cette interprétation que la jeune femme s'appropria, elle exposa d'elle-même, et ce dès l'introduction de sa demande d'asile, une phrase concise qui évoquait les menaces de Jérôme. On peut penser que ce chantage avait probablement contribué à ce qu'elle efface, en partie, sa trajectoire singulière au profit de la sienne. 


\section{Une reconstruction narrative}

Anna, en s'appropriant les éléments douloureux de son passé, put approcher quelque chose des déformations qui avaient engendré des versions testimoniales multiples, dont la fonction était protectrice et défensive. Le lent et long processus d'élaboration et de déconstruction des versions précédentes liées à ses premières déclarations l'autorisa à construire une version plus subjectivée. Une « resignification du passé » semblait s'opérer avec le travail dereconstruction narrative que nous avions mis en place [4]. Les événements de son histoire individuelle s'agençaient à ceux de l'histoire collective et réintroduisaient une temporalité signifiante pour elle.

Lorsque Anna sentit qu'elle approchait de la fin de la rédaction de son récit, l'épreuve d'écriture à deux s'arrêta. Elle dut adjoindre à son dossier destiné à l'Ofpra un certain nombre de preuves confirmant l'authenticité de ses propos (attestation de suivi psychologique, attestation de l'école concernant ses « bons résultats », etc. $)^{9}$.

Puis, peu après son audition avec un agent de l'État, Anna reçut un document stipulant sa qualité de réfugié. Il est intéressant de remarquer qu'aucun délai d'attente entre cette entrevue et la délivrance du statut n'eut lieu : la réponse administrative advint avant même que le délai annoncé n'ait atteint son terme. Si cette réponse immédiate renvoie probablement, et en partie, à des accords politiques transversaux, cette levée du soupçon témoigne à mon sens de plusieurs points. D'une part, des effets sur l'interlocuteur institutionnel d'un sujet qui fait preuve d'une narration habitée et structurée et d'un récit qui comporte peu de zones d'ombres. D'autre part, de l'importance des preuves et des « expertises » adjointes à son dossier (attestations diverses) venues appuyer et «valider» sa requête.

Je pense que le suivi psychothérapeutique, conjugué au travail d'écriture à deux, n'est guère étranger à l'obtention de son statut, la fonction du psychologue favorisant la reprise de la capacité narrative du sujet. L'organisation du récit empêchée par les effets du traumatisme se remet progressivement en mouvement avec l'émergence d'une parole singularisée sur la scène transférentielle.

Anna avait saisi rapidement l'investissement dont elle faisait l'objet par les membres de l'association, et en particulier mon désir de la soutenir dans son projet de « renar-

\footnotetext{
${ }^{9}$ En tant que psychologue, je lui proposai une « attestation de suivi psychologique ». Ce document allait dans le sens d'une « validation » de son témoignage qui m'amenait finalement à prendre partie et à appuyer sa demande d'asile auprès de l'État. Après-coup, là encore, la thématique des attestations délivrées au patient et destinées aux instances décisionnelles suscita nombre de questionnements en moi. Je m'étais « collée » au discours de l'État : « faire », « expertiser », entrant ainsi, et de manière paradoxale, dans le système d'instrumentalisation imposé par l'institution juridique, tout en restant prise dans cette posture d'engagement associatif. La crainte qui m'amena à agir et à sortir de ma neutralité reposait sur le fait que je redoutais un possible rejet qui signerait son déboutage définitif et probablement par là-même... la chute de mes idéaux humanistes !
}

ration ». En raison de sa capacité psychique à reconstruire son histoire fragmentée et à redonner de l'ordre aux événements vécus mais également à investir un espace humain où il lui fut possible de trouver des témoins garants, elle put s'inscrire dans une demande à l'Autre, ciblée, et à utiliser habilement les différents lieux qui lui étaient proposés - avec les psychologues, l'appui d'associatifs, son avocate, l'assistante sociale de la ville et de son école, etc.

Le déploiement de ses ressources internes pour obtenir l'asile ne manifestaient-ils pas le retour à une certaine mobilité psychique ? Accueillir sa demande d'accompagnement narratif n'a-t-il pas favorisé chez elle la reprise d'une position plus active par rapport aux événements traumatiques ? Le temps de sidération du traumatisme semblait bien toucher à sa fin, lui permettant de sortir progressivement de son destin mortifère.

\section{L' " après-coup » de l'annonce de l'accord institutionnel}

Je proposai à Anna une entrevue, trois mois après l'obtention de sa qualité de réfugié, afin de percevoir la façon dont elle accueillait l'arrivée de son nouveau statut. Ce rendez-vous qui fut difficile à fixer marquait le retrait et le désinvestissement de notre relation par cette jeune femme et mettait en relief divers points. Bien qu'ayant vécu des élans transférentiels massifs à mon égard, Anna avait déposé des souvenirs et des souffrances auxquels j'étais désormais identifiée. Ne tentait-elle pas, en repoussant cet entretien, de se dégager du dépôt de maux effectué auprès de moi ?

Tout en s'étayant sur ma position de clinicienne, elle avait intégré ma fonction de passeur, «temporaire » et intermédiaire. Elle finit par venir à l'entretien et me dit ce jour-là : "Je suis heureuse de l'avoir [le statut de réfugié],

j'ai fait une grande fête... Mais maintenant tout est compliqué. » Il lui fallait en effet quitter le foyer, trouver un emploi et payer un logement. L'euphorie des premiers instants laissait place à une réalité plus difficile, qu'elle semblait néanmoins à même « négocier » sans s'effondrer.

Anna me raconta qu'elle était désormais en psychothérapie avec une femme, son thérapeute l'ayant spontanément orientée vers une collègue. «Il a senti » que le travail auprès d'une psychothérapeute serait plus porteur. Et elle ajouta " mais même avec elle, je n'arrive pas à oublier le passé, à en parler... », comme pour me protéger de ce nouvel investissement, garder quelque chose du lien privilégié qui s'était créé entre nous.

Anna put donc « rebondir » en investissant un autre espace psychothérapeutique. Son nouveau statut impliquait peut-être pour elle la possibilité d'une existence autre et d'une nouvelle thérapeute.

Plusieurs mois après, et «par hasard », à l'occasion d'une manifestation culturelle, je la rencontrai. Elle sem- 
blait épanouie, avait rencontré un petit ami, et souhaitait reprendre la danse, qu'elle appréciait beaucoup enfant. Elle me remercia ; notre rencontre s'acheva sur cette entrevue impromptue.

\section{Les questionnements induits par cette rencontre}

Ce parcours à deux qui s'est étayé sur une écriture en souffrance pose un certain nombre de problèmes. Quels sont les effets du témoignage du survivant sur son interlocuteur et sur le clinicien en particulier ? Comment le soignant peut-il «négocier » la politique du témoignage instrumentalisé et s'en dégager au sein de l'espace clinique qu'il propose?

Elle introduit également la question de la symptomatologie post-traumatique, et notamment la mémoire « trouée » et ses incidences sur la perte des repères spatiotemporels, le verrouillage de certaines scènes, et leurs affects liés qui empêchent la remémoration. La fonction du « récit écran » qui masquerait une réalité inénarrable est ici posée et débouche sur ce que les autorités considèrent finalement comme un «faux témoignage » [8].

Apparaissent également les liens entre la mise en récit et la réactualisation d'une trajectoire traumatique enracinée dans une histoire infantile refoulée ainsi que le travail de reconstruction par le biais de l'espace transférentiel.

La rencontre avec Anna est à mon sens symptomatique des difficultés inhérentes au travail clinique conduit par les psychothérapeutes auprès d'une population réfugiéesurvivante. Le débordement des affects, le vacillement de

la neutralité ainsi que l'entrée massive dans l'agir, à laquelle j'ai cédé, introduisent directement la puissance du transfert qui se déploie chez le clinicien, phénomène à la mesure de l'intensité de celui du patient. La possibilité

même qu'Anna puisse être déboutée du droit d'asile, annonce souveraine impliquant une non-reconnaissance par le politique de ses persécutions, provoquait en moi une crainte terrible : qu'elle se sente, à nouveau, abandonnée

par la communauté humaine. L'administratif exigeant entra en écho avec mes propres peurs et me conduisit à l'agir : aider Anna à composer son récit, lui proposer une attestation psychologique, être un témoin actif du témoin.

On voit ainsi se dessiner les effets psychiques produits par une telle « injonction souveraine à relater » sur le rescapé et le psychologue. Les croisements, voire les confusions entre réalité interne et externe, scène juridique et psychique, se profilent comme caractéristiques de cette clinique particulière.

Si la posture que j'adoptais est critiquable à bien des égards, augurant d'un glissement entre la clinicienne et la femme engagée que je suis, cette expérience s'avère paroxystique des entremêlements qu'une telle clinique est susceptible d'engendrer.
L'《 aide clinique » au témoignage s'écarte de la posture clinique et du travail psychothérapeutique, puisqu'elle encourage, d'une manière ou d'une autre, " à faire dire » son vécu au réfugié. Pour autant, doit-on, à partir de cette expérience, parler d'un « forçage » du discours du sujet, d'une instrumentalisation de la clinique ? Ce travail de mémoire et de reconstruction narrative ne se rapproche-t-il pas davantage de la position du « témoignaire ${ }^{10}$ que de celle du clinicien?

La figure de témoignaire se distingue de celle du clinicien ou de l'analyste, dans la mesure où il s'inscrit dans une « interaction facilitante $»^{11}$ et qu'il favorise un travail de tissage textuel. L'éthique de cet interlocuteur l'amène à respecter la temporalité du sujet et à ne pas le «presser» pour en savoir plus.

Combler les lacunes d'une mémoire traumatique en souffrance, telle était la tâche à laquelle je m'étais engagée avec Anna. Et je restai attentive à ce que ce cocheminement puisse se réaliser sans me «coller » à une logique de la pièce à conviction, d'une vérité à tout prix, le travail de mise en récit reposant avant tout sur sa propre capacité élaborative et associative. Avec sa psychothérapie menée parallèlement, les prémices d'un après-coup du trauma cheminaient probablement déjà chez cette jeune femme «prête » à effectuer un travail de mise en mots. Je la rencontrai dans un moment fécond, alors qu'elle était en capacité d'entrer dans un processus d'historicisation et de « faire quelque chose » de sa trajectoire chaotique.

À partir de la création d'un lieu et d'un dispositif singuliers, un lent processus de co-construction put se déployer par le truchement de la scène transférentielle. La mise en récit d'Anna s'est effectuée dans une perspective de travail

« cosubjectif », comme l'envisage R. Waintrater lorsqu'elle s'intéresse à la relation témoignaire/témoin. Il s'est ainsi opéré un travail de reconstruction du témoignage dans la visée d'une «reconstitution historienne » [6]. Si cette co-élaboration favorisa l'avènement d'une narration mobile et subjectivée, c'est aussi sans doute parce que, dans le même temps, ce processus favorisa une «mise en terre » des siens [1]. La partielle symbolisation des événements par l'avènement des mots permit la naissance d'un récit qui fut structuré par la transformation de « traces a-mnésiques » en traces mnésiques puis en représentations, et ce grâce au pouvoir symboligène que revêt l'écriture. La manière dont Anna put s'immerger, investir la langue française, par le biais de notre rencontre, contribua aussi à ce qu'elle produise un discours véridique. Elle trouva en effet dans cette langue l'hospitalité, le refuge en vain recherché.

Favoriser la mise en mots d'une trajectoire douloureuse en vue de l'obtention d'un statut s'écarte fondamentalement de la perspective clinique et soulève la question des

\footnotetext{
${ }^{10}$ R. Waintrater (2003). Sortir du génocide. Op. cit, p. 221 [13].

${ }^{11}$ R. Waintrater. Op. cit., p. 223 [13].
} 
glissements, voire de la collision potentielle qui réside entre les champs du juridique, du psychique, du médical et du social pour le praticien confronté à la survivance. Car la scène judiciaire, omniprésente dans ce champ clinique est toujours en toile de fond, et susurre au sujet ainsi qu'à tous les interlocuteurs avec qui il entre en contact : « Raconte tout ! » Impératif de terreur inhérent aux règles instituées par le système judiciaire. Il apparaît donc essentiel que le clinicien ait conscience de l'influence de ce discours pour qu'il soit en mesure de ne pas s'y conformer, et que soient ainsi garanties sa posture et sa neutralité. C'est à cette condition que la temporalité interne du sujet ne sera pas substituée par le temps judiciaire.

Des avancées de l'ordre du thérapeutique se sont produites chez Anna dans la mesure où son état psychique s'est amélioré au fil des entrevues, et ce parallèlement au contexte extérieur qui évoluait positivement. Et même s'il est naïf de penser « elle a obtenu son statut de réfugié et elle va mieux », on perçoit la façon dont la réalité psychique et les réponses environnementales interagissent considérablement.

Le cas d'Anna permet d'observer les effets relatifs à la tentative de déshumanisation des tortionnaires sur le sujet mais aussi son déplacement sur l'interlocuteur. On perçoit comment le clinicien se « raccroche » à l'environnement et surinvestit la matérialité pour se protéger des attaques du Réel, transmises par le transfert massif du sujet rescapé.

Si l'issue transférentielle et matérielle fut ici « heureuse », elle met en relief l'investissement commun entre patient et clinicien autour d'un objet réel - le certificat de réfugié - et amène à penser le danger impliqué par cet investissement dirigé sur un objet extérieur, puisqu'il est, précisément, susceptible de ne pas être satisfait et donc de provoquer l'effondrement du patient et du thérapeute. La matérialité des «papiers » est en quelque sorte venue gratifier l'interlocutrice désemparée que j'étais face à cette jeune rescapée, et la détresse ressentie trouva une forme d'issue partielle à travers cet objet. Le « couronnement narcissique » provoqué par l'acceptation du statut aurait cédé la place au dépit et à l'impuissance si ce rejet souverain était advenu ; ces sentiments auraient alors pu entrer en miroir avec ceux de la patiente et auraient conduit à un possible redoublement de détresse. Là, dans cette situation précise, les temps psychique et judiciaire se sont emboîtés, alors qu'ils entrent le plus fréquemment en collision lors du parcours administratif des réfugiés.

Il fallut « de l'Autre » à Anna, garant et fiable, à qui elle puisse s'adresser pour être en mesure de resubjectiver son histoire, et c'est par l'établissement d'un lien transférentiel qu'un corpus écrit, plus subjectivé, put éclore. L'historicisation progressive de sa trajectoire lui a permis d'être dans l'élection d'une version qu'elle signa en son nom propre.

Le chevauchement, la captation des événements récents, si violents par le traumatisme sexuel, ont favorisé l'assemblage des fragments de son histoire où des éléments exclus et d'autres refoulés cohabitaient. On retrouve ici la structure du trauma avec le premier temps et le second temps qui vient le révéler. C'est en associant d'elle-même et en effectuant des passerelles au sein de son histoire que la narrativité a pu s'arracher progressivement de son opacité et qu'un témoignage structuré a pu se tisser. Le travail psychothérapeutique, d'une part, et l'écriture dans la langue française, d'autre part, contribuèrent probablement à ce que la jeune femme produise une version singularisée de sa trajectoire. À partir de cette rencontre clinique et d'autres qui ont suivi, l'expression " clinique de l'asile »" permettrait de penser cette praxis complexe qui se situe aux limites des souffrances humaines $[3,11]$.

\section{Références}

1. Altounian J. La Survivance. Traduire le trauma collectif. Paris : Dunod, coll. «Inconscient et culture ». L'introduction de cet ouvrage s'intitule « Mettre en mots, mettre en terre, se démettre des ancêtres », 2000 ; (p.1).

2. Antelme R. (1947). L'Espèce humaine. Paris : TEL Gallimard, 1957.

3. Benslama F. Clinique de l'exil. Cahiers Intersignes. 2000 : $14-5$.

4. Braunstein N. La Jouissance, un concept lacanien. Ramonville-Saint-Agne : Érès, « Point Hors Ligne », 2005 ; (p.169).

5. Chasseguet-Smirgel J. Construction en analyse et construction psychique. In Revue française de Psychanalyse. In : " Mémoire et souvenir dans la théorie et la pratique psychanalytique », tome XLIII. Paris : PUF, 1979 : 655 ; $(664,665)$.

6. Chiantaretto JF. L'écriture. Cours de D.E.S.S. de sciences humaines cliniques, Paris-VII-Denis-Diderot, 2000-2001.

7. Devereux G. De l'angoisse de la méthode dans les sciences du comportement. Paris : Flammarion, « Nouvelle bibliothèque scientifique », 1980.

8. FREUD S. In : (1899). «Sur les souvenirs écrans ». In Névrose, psychose et perversion. Paris : PUF, 1988.

9. De Mijolla-Mejor S. Dictionnaire international de psychanalyse, t. II. Paris : Calmann-Levy, 2002 ; (sous la dir. d'A. de Mijolla).

\footnotetext{
${ }^{12}$ Cette expression renvoie à l'entité « clinique de l'exil », paradigme créé par les auteurs F. Benslama, O. Douville, A. Cherki, L. Tourn, etc., dans les années 1990. Lire notamment l'ouvrage de référence dirigé par F. Benslama, « Clinique de l'exil ». Cahiers Intersignes $2000 ; 14-15$.
} 
10. KIRMAYER L. The refugee's predicament. Traduction française de Bouville JF, Rechtman R, Le dilemme du réfugié. Victimes 2002 ; 67 : 743-63.

11. Pestre É. «L'État, le réfugié et son thérapeute. Les conditions de vie psychiques des réfugiés ». Thèse soutenue le 23 juin 2007, université Paris-VII, p. 238-338.
12. Rousseau C. Uncertainty and transcultural clinical pratice («Incertitude et clinique transculturelle »). Evol Psychiatr $2002: 764-74$

13. WAINTRATER R. Sortir du génocide. Témoigner pour réapprendre à vivre. Paris : Payot, 2003. 